\title{
CANAIS \\ UNIVERSITÁRIOS DE TV A CABO - TV USP
}

A realidade vem por força da lei.

Para cumprir o que determina a Lei de televisão a cabo (Lei n. 8977 de 06/01/95), multiplica-se a criação dos Canais Universitários, nos municípios brasileiros que possuem universidades.

A emissão é municipal, isto é, o alcance das imagens de cada canal restringe-se ao município em que estão sediadas as reitorias das universidades.

Para cumprir e garantir esse direito, o território brasileiro começa a cobrir-se de Canais Universitários de TV.

Dúvida, descrença, perplexidade, desconfiança ... mas também, claro, algum entusiasmo.

Esse último está mais com aqueles que aceitaram o desafio e arregaçaram as mangas para inventar essas novas TVs. O espírito dos pioneiros apóia-se na certeza de que neste fim de década/século/milênio a comunicação de qualquer coisa fica mais sólida pela via da telinha.

Observa-se também, e isso muito me alivia, que a forma dessa comunicação busca seus modelos na linguagem da TV - por mais simples que seja o formato - deixando de lado o estilo sala de aula. Entendo isso como promissor.

\section{A AUTORA}

\section{Marília Franco}

Professora Doutora do Departamento de Cinema, Rádio e Televisão da Escola de Comunicações e Artes e Coordenadora Acadêmica da TV USP.

E-mail: mdsfranco@usp.br
O que se vê, no entanto, ainda é muito próximo do institucional/ propaganda. Quase um venha pra Uni... qualquer coisa... você também.

Vamos compreender a tentação. Afinal todos os canais comerciais nos ensinam, desde a década de 50, esse estilo de produção de TV.

Como não é fácil ocupar diariamente um canal de televisão, por cinco minutos que seja, as universidades de cada município estão se organizando em grupos - consórcios - e entrando em acordos quanto à forma de ocupar os horários, adquirir equipamentos em comum, redigir e cumprir códigos de ética de programação.

Estão entendendo o significado da liberdade responsável na ocupação de cada horário. Estão definindo formas de patrocínio e, com isso, criando uma nova realidade comercial de tempo de TV.

Alguém duvida de que se cria um novo território de convivência entre instituições que, até hoje, disputaram espaço na conquista das melhores cabeças?

\section{PRÓS E CONTRAS}

Vamos começar com os contras.

$\mathrm{O}$ primeiro argumento é de que não fica clara a relação custo/benefício para as universidades.

Explicando melhor: fazer televisão é muito caro. $\mathrm{O}$ ensino universitário, de um modo geral, passa por uma crise de custos, entre outras. 
Para as universidades públicas a situação está quase insustentável, pois o Estado investe cada vez menos em ensino superior e em pesquisa. Para as escolas particulares o custeio através das mensalidades tem que restringir-se aos limites do suportável para o bolso dos alunos.

Como sustentar então um gasto complexo e alto como montar uma produtora de TV dentro das universidades ou contratar terceiros para gerar programação?

Seria hora dessa aventura?

Falando de dentro do problema afirmo que a hora é agora. Em crise estamos sempre, portanto não dá para superar crises para depois inovar. Em qualquer coisa que seja.

Outro contra fica por conta do temor de conteúdo.

$\mathrm{O}$ que as universidades têm de tão interessante para contar pela televisão? Não ficará uma mesmice de entrevistas e debates sobre os mesmos temas globais (de globalização)?

Talvez sim, no começo. Mas sempre será interessante acompanhar o esforço de reflexão sobre a realidade, por parte da comunidade, instituída pela sociedade para produzir pensamento inteligente e/ou conhecimento.

E neste ponto quero começar a argumentar a favor.

Em primeiro lugar é preciso entender que resultados, na inovação de procedimentos, não serão sólidos se buscados através de parâmetros imediatistas.

A TV comercial mede seu valor por índices de audiência - número de aparelhos ligados naquele momento. $E$ a relação custo/benefício reflete-se no valor do minuto para veicular publicidade (o que paga o custo da produção dos programas).
Do ponto de vista cultural essa articulação mostra-se, algumas vezes, um desastre. Sensacionalismos de todo tipo, violência, pornografia, baixarias de um modo geral, mais vezes do que o desejado, elevam a relação custo/benefício da TV comercial.

Essa realidade modifica-se bastante no universo das TVs segmentadas, aqueles canais cuja programação é dirigida a públicos e interesses específicos (compras, filmes, desenhos, viagens, notícias, esportes, religiões etc.).

Esse é o universo das TVs a cabo, TVs por assinatura ou pay-per-view (mais ou menos: pague apenas pelo que viu).

É também o universo dos canais de interesse específico de segmentos geográficos - Canal Comunitário, Canal Universitário, Canal Legislativo, sempre com alcance municipal.

Pode-se perceber, portanto, que a avaliação da relação custo/benefício precisa ser calculada sob outros parâmetros.

Seguindo a prática em países de primeiro mundo, começa a haver no Brasil uma modificação significativa na distribuição das verbas aplicadas em cultura.

Até a década passada o patrocínio das atividades culturais e artísticas, de modo geral, estava quase restrito aos concursos, premiações ou verbas do Estado. Já no fim dos anos 80 , no entanto, a Lei Sarney introduziu modificações interessantes nesse panorama, estimulando a iniciativa privada a aplicar em cultura, através de incentivos fiscais.

Quando os primeiros efeitos começariam a ser sentidos veio a era Collor que desmanchou tudo sem pôr nada no lugar. Retomada a questão, desde o governo Itamar Franco, as leis federais vêm sendo ampliadas e aperfeiçoadas, estimulando a criação de legislações estaduais e municipais que hoje clareiam o horizonte das relações entre empresas e produtores culturais. 
Essas possibilidades, mais uma vez aproveitadas por força de lei, vêm ajudando a desenvolver uma cultura de patrocínio entre os empresários tanto quanto consolidando uma saudável atitude de administrar a produção cultural e artística sob a ótica da qualidade/lucro.

Essas mudanças todas favorecem a criação de interesse de patrocínio para TVs segmentadas como os Canais Universitários, reforçada pela prática bem-sucedida dos apoios culturais à programação das TVs educativas, como a TV Cultura.

Além desse lado mais pragmático das relações comerciais entre patrocinadores e produtores culturais, é preciso analisar o valor do tipo de bem que as universidades produzem e podem veicular num canal de televisão - informação, ciência, cultura, arte.

Educação continuada e a distância é um termo emergente e urgente. Os americanos cunharam uma palavra síntese para a produção de materiais que favoreçam a construção do conhecimento aliada ao prazer: edutainment, de education + entertainment (educação e entretenimento).

$\mathrm{O}$ investimento em cultura e educação só pode ser planejado para avaliação de resultados a médio e longo prazo. Os empresários começam a entender isso e, principalmente, começam a compreender o quanto esse tipo de investimento reverte em benefício da formação de um trabalhador mais cidadão e, como consequiência, mais útil ao desenvolvimento da sociedade.

Os exemplos mais claros dessa mudança de mentalidade e atitude é o investimento das empresas em grandes projetos de televisão como Telecurso 2000 e Canal Futura. Com resultados em prazos mais curtos do que os esperados.

Mesmo que nos ventos de uma lei não emanada da luta das universidades, a oportunidade tem que ser aproveitada agora. No âmbito externo às universidades o panorama é favorável à ocupação desse espaço.

Olhando para dentro do mundo acadêmico o momento torna-se mais grave, premente e oportuníssimo. A formação cultural dos indivíduos já se faz há tantas décadas por via da informação audiovisual. Espectadores somos todos. Experts em espectar a visão do mundo que as vozes e olhos da mídia autorizada nos trazem à mesa do jantar. $\mathrm{E}$, no entanto, as escolas (primeiro, segundo e terceiro graus) continuam modelando nossa expressão através do discurso oral e escrito.

Frenéticos consumidores dos discursos audiovisuais, ainda mantemos o mito de que a expressão audiovisual é privilégio de poucos eleitos.

Sei na pele o rebuliço que causa propor ao mestre/pesquisador que exponha seu conhecimento com outros recursos que não o texto e/ou a aula. Todos os que aceitam e vencem esse desafio percebem o quanto ganham ampliando seu horizonte expressivo.

Os Canais Universitários abrem o mais precioso espaço de ampliação desse exercício de atualização do discurso acadêmico. Sem esse mergulho no mundo contemporâneo das mídias, a palavra da ciência estava correndo o risco de virar um dialeto em extinção.

A cautela e a responsabilidade com que as universidades vêm se irmanando na inauguração dessa nova prática de comunicação deixa-me otimista quanto aos resultados. Mas a relação custo/benefício é muito difícil sequer de prever, pois os parâmetros são novos demais para autorizar prognósticos.

Curiosidade, experimentação, liberdade e otimismo podem ser uma orientação mais segura para essa aventura indispensável do que um embolorado espírito de cautela crítica frente a esse desafio por força da lei. 


\section{UM PEQUENO RELATO - A TV USP}

A USP - Universidade de São Paulo faz parte do grupo das nove universidades do município de São Paulo que, desde 10 de novembro de 1997, puseram no ar o $C N U$ Canal Universitário. As outras oito são:

PUC - Pontifícia Universidade Católica (pioneira na experiência), UM Universidade Mackenzie, UNIBAN Universidade Bandeirante, UNICSUL Universidade Cruzeiro do Sul, UNIFESP Universidade Federal de São Paulo/Escola Paulista de Medicina, UNIP - Universidade Paulista, UNISA - Universidade Santo Amaro, Universidade São Judas.

Para a USP não foi uma decisão fácil aderir ao consórcio, mas também não seria desejável que ficasse de fora do projeto, adiando a oportunidade de ampliar seu espaço de comunicação com a sociedade.

Assim ficou decidido que a USP abrigaria sua TV dentro da CCS - Coordenadoria de Comunicação Social, na companhia do Jornal da USP, Rádio USP, USPon-line e outras atividades de comunicação interna e externa.

Na elaboração do projeto da TV USP duas perguntas nortearam a reflexão:

- que imagem da USP queremos transmitir?

- que compromisso de diálogo assumiremos com os espectadores?

A busca das respostas foi sempre orientada pela consciência de que a universidade pública tem um compromisso com a população que a custeia através dos impostos.

Por outro lado a USP já tem uma imagem construída ao longo dos seus quase 64 anos de existência. Um nome respeitável e respeitado. A experiência de expor-se numa mídia nova, e não afeita ao espaço da docência e da pesquisa, merece uma atenção redobrada.
Assim, para responder a primeira pergunta, combinaram-se os conceitos consagrados da USP com as responsabilidades do novo projeto, apoiando a busca dessa imagem em:

- pluralismo,

- excelência,

- interatividade,

- valorização da construção do conhecimento,

- formação cultural das novas gerações,

- resposta ao investimento da sociedade.

Já a segunda pergunta exigia que definíssemos se nossos espectadores seriam nosso público interno, isto é, alunos, docentes e funcionários ou se abriríamos o espaço ao diálogo com a sociedade fora do campus.

Ficamos com a segunda opção, inclusive porque a USP já tem vários mecanismos internos de comunicação, ainda não completos, mas em permanente ampliação.

Ao definir, no entanto, seu públicoalvo - a sociedade - a TV USP tem plena consciência de que essa postura "assume dimensões muito especiais, considerado o compromisso da Universidade pública com a construção e transmissão do conhecimento na sua dimensão de excelência e utilidade".

"Os mecanismos de feed-back da TV USP terão que ser construídos segundo a finalidade social mais elevada da universidade e não a partir de desejos e modismos mensuráveis por métodos tradicionais de aferição de audiência.

"Essas duas direções conceituais definem, portanto, o papel da TV USP não como uma vitrina de exibição das qualidades acadêmicas, mas sobretudo como a possibilidade de resgate da solidariedade, do respeito e do desenvolvimento mútuo em que devem se pautar as relações entre os produtores do saber e a 
sociedade que os inspira e os financia.

"Para que esses conceitos sejam consolidados nas ações práticas da produção dos programas, é preciso que alguns princípios sejam estabelecidos como norteadores do tratamento dos projetos de programação e da avaliação de qualidade dos produtos:

- a linguagem de TV terá primazia sobre qualquer outro formato;

- a informação será transmitida a leigos e não a iniciados;

- a TV deverá transmitir a voz de todos os segmentos acadêmicos" (trecho do projeto da TV USP).

Uma vez definidos conceitos e princípios, mãos à obra!

E, claro, toda obra complexa merece ser construída com base e cautela.

A base partiu dos arquivos audiovisuais da USP, organizados dentro da CECAE - Coordenadoria Executiva de Cooperação Universitária e Atividades Especiais através do Univídeo, projeto de fomento ao desenvolvimento das atividades audiovisuais na universidade e com o apoio técnico do SIBI - Sistema Integrado de Bibliotecas.

O Univídeo foi cadastrando, desde o início de suas atividades em 1993, filmes e vídeos produzidos para o ensino, a pesquisa e a extensão de todas as unidades da USP. Esse catálogo mostrou-nos uma face audiovisual que desconhecíamos, por causa da dispersão dessa informação.

Quando se configurou a criação da $T V$ USP esse banco de dados tornou-se um precioso apoio à viabilização de uma grade de programação. Nestes quase três meses de transmissão é com ele que temos alimentado nossas emissões.

A cautela nos é imposta, antes de mais nada, pelo ritmo tradicionalmente lento da USP em abraçar as inovações. Confesso que às vezes me aflijo para conciliar essa natureza acadêmica às exigências de pressa da produção de TV. Tudo isso aliado ao ritmo administrativo do Estado e suas regras burocráticas.

A estrutura da $T V U S P$, no entanto, está montada e andando. As parcerias internas - com a ECA - Escola de Comunicações e Artes e com a FAU Faculdade de Arquitetura e Urbanismo, entre outras unidades, vêm mostrando que só a mobilização conjunta poderá garantir que a imagem da USP será plural, na medida do esforço e compromisso de toda a comunidade.

Nosso modelo de produção parte desses princípios. Não queremos terceirizar nem centralizar a produção, sobretudo não queremos centralizar a criação de projetos.

Aos poucos outras parcerias começam a se configurar e podemos vislumbrar uma grade de programação que venha a realizar o que está definido no projeto da TV USP.

Também parcerias de produção com nossas companheiras de consórcio começam a ser desenhadas.

Assim abraçamos esta aventura indispensável com curiosidade, experimentação, liberdade e otimismo. Só essa consciência e esse estado de espírito podem sustentar o desafio.

A partir de março começamos a veicular programas realizados inteiramente pela equipe da TV USP e abrimos o convite à participação de toda a comunidade acadêmica na apresentação de projetos e produtos. Do mesmo modo esperamos a manifestação de nossos espectadores para que a interatividade comece a orientar a direção de nossa produção.

O CNU - Canal Universitário vai ao ar pelo canal 15 da NET e Multicanal ou canais 67 e 68 da TVA. 


\section{CNU - Canal Universitário - Grade de Programação ${ }^{1}$}

\begin{tabular}{|c|c|c|c|c|c|c|c|}
\hline Dia & Segunda & Terça & Quarta & Quinta & Sexta & Sábado & Domingo \\
\hline $8: 00$ & USP & S.JUDAS & UNISA & PUC & UNICSUL & UNIFESP & UNIP \\
\hline $8: 30$ & MACK & USP & S.JUDAS & UNISA & PUC & UNICSUL & UNIFESP \\
\hline 9:00 & UNIP & MACK & USP & S.JUDAS & UNISA & PUC & UNICSUL \\
\hline $9: 30$ & UNIFESP & UNIP & MACK & USP & S.JUDAS & UNISA & PUC \\
\hline $10: 00$ & UNICSUL & UNIFESP & UNIP & MACK & USP & S.JUDAS & UNISA \\
\hline $10: 30$ & UNIBAN & UNIBAN & UNIBAN & UNIBAN & UNIBAN & UNIBAN & UNIBAN \\
\hline 11:00 & PUC & UNICSUL & UNIFESP & UNIP & MACK & USP & S.JUDAS \\
\hline $11: 30$ & UNISA & PUC & UNICSUL & UNIFESP & UNIP & MACK & USP \\
\hline $12: 00$ & S.JUDAS & UNISA & PUC & UNICSUL & UNIFESP & UNIP & MACK \\
\hline $12: 30$ & USP & S.JUDAS & UNISA & PUC & UNICSUL & UNIFESP & UNIP \\
\hline $13: 00$ & MACK & USP & S.JUDAS & UNISA & PUC & UNICSUL & UNIFESP \\
\hline $13: 30$ & UNIP & MACK & USP & S.JUDAS & UNISA & PUC & UNICSUL \\
\hline $14: 00$ & UNIFESP & UNIP & MACK & USP & S.JUDAS & UNISA & PUC \\
\hline $14: 30$ & UNICSUL & UNIFESP & UNIP & MACK & USP & S.JUDAS & UNISA \\
\hline $15: 00$ & UNIBAN & UNIBAN & UNIBAN & UNIBAN & UNIBAN & UNIBAN & UNIBAN \\
\hline $15: 30$ & PUC & UNICSUL & UNIFESP & UNIP & MACK & USP & S.JUDAS \\
\hline 16:00 & UNISA & PUC & UNICSUL & UNIFESP & UNIP & MACK & USP \\
\hline $16: 30$ & S.JUDAS & UNISA & PUC & UNICSUL & UNIFESP & UNIP & MACK \\
\hline $17: 00$ & USP & S.JUDAS & UNISA & PUC & UNICSUL & UNIFESP & UNIP \\
\hline $17: 30$ & MACK & USP & S.JUDAS & UNISA & PUC & UNICSUL & UNIFESP \\
\hline $18: 00$ & UNIP & MACK & USP & S.JUDAS & UNISA & PUC & UNICSUL \\
\hline $18: 30$ & UNIFESP & UNIP & MACK & USP & S.JUDAS & UNISA & PUC \\
\hline 19:00 & UNICSUL & UNIFESP & UNIP & MACK & USP & S.JUDAS & UNISA \\
\hline $19: 30$ & UNIBAN & UNIBAN & UNIBAN & UNIBAN & UNIBAN & UNIBAN & UNIBAN \\
\hline 20:00 & PUC & UNICSUL & UNIFESP & UNIP & MACK & USP & S.JUDAS \\
\hline $20: 30$ & UNISA & PUC & UNICSUL & UNIFESP & UNIP & MACK & USP \\
\hline 21:00 & S.JUDAS & UNISA & PUC & UNICSUL & UNIFESP & UNIP & MACK \\
\hline $21: 30$ & USP & S.JUDAS & UNISA & PUC & UNICSUL & UNIFESP & UNIP \\
\hline 22:00 & MACK & USP & S.JUDAS & UNISA & PUC & UNICSUL & UNIFESP \\
\hline $22: 30$ & UNIP & MACK & USP & S.JUDAS & UNISA & PUC & UNICSUL \\
\hline 23:00 & UNIFESP & UNIP & MACK & USP & S.JUDAS & UNISA & PUC \\
\hline $23: 30$ & UNICSUL & UNIFESP & UNIP & MACK & USP & S.JUDAS & UNISA \\
\hline 00:00 & UNIBAN & UNIBAN & UNIBAN & UNIBAN & UNIBAN & UNIBAN & UNIBAN \\
\hline $00: 30$ & PUC & UNICSUL & UNIFESP & UNIP & MACK & USP & S.JUDAS \\
\hline 01:00 & UNISA & PUC & UNICSUL & UNIFESP & UNIP & MACK & USP \\
\hline
\end{tabular}

1. Grade de programação que vigora desde 9 de fevereiro de 1998. 
Resumo: $\mathrm{O}$ artigo destaca a oportunidade da criação de canais universitários, através da Lei de televisão a cabo, abrindo à sociedade a veiculação da programação de nove universidades paulistas. Destaca o desafio em que consiste o meio acadêmico utilizar-se da linguagem televisual na sua relação com a sociedade. A TV USP, como membro do $C N U$, tem-se pautado pelos conceitos de construção e transmissão de conhecimento na sua dimensão de excelência e utilidade, buscando parcerias internas entre suas unidades para a veiculação de programação. A autora divulga grade de programação que entrou em vigor em 9/2/98.

Palavras-chave: Lei de televisão a cabo, canal universitário, TV USP, universidade, cultura, ciência
Abstract: The article stresses the opportunity of creating university stations, via the cable television Law, opening society to the transmission of neuf universities of the State of São Paulo. It emphasizes the challenge it represents to the academic realms to use the television language in its relationship with society. TV USP, as a CNU member, has guided itself by the concepts of knowledge construction and transmission in its dimension of excellence and utility, searching from internal partnerships between its units for transmitting its programming. The author divulges the programming that is effective as of $2 / 9 / 98$.

Key words: Cable television Law, university station, TV USP, university, culture, science 